\title{
Electrocardiographic Parameters Indicative for Increased Risk of Adverse Events in Diabetics after Coronary Artery Bypass Grafting
}

\author{
Dimitar Simov $^{1}$, Ivaylo Christov ${ }^{1}$, Iana Simova ${ }^{2 *}$, Mikhail Matveev $^{1}$, Ivo Petrov ${ }^{2}$ \\ ${ }^{1}$.Institute of Biophysics and Biomedical Engineering, Bulg. Acad. of Sci, Sofia, Bulgaria \\ ${ }^{2}$.Acibadem City Clinic, Cardiovascular center - University Hospital, Sofia, Bulgaria
}

\begin{abstract}
Objectives: The goal of the study is to analyze the changes of ECG parameters caused by coronary artery bypass grafting $(C A B G)$, and to evaluate their relevance to the assessment of cardiac risk in diabetics (DM) and nondiabetic patients

Materials and Methods: ECG recordings of 50 patients (men 48, mean age 63.2 \pm 7.5) undergoing CABG surgery were collected: The number of bypass-per patient varied from 1 to 4 (47\% of patients received 4). ECGs were recorded pre-and post-surgery, from 2 to 10 days after the intervention. Eighteen patients were diagnosed with type II diabetes. Six of these diabetics were on subcutaneous insulin treatment.
\end{abstract}

Results and Discussions: The parameters' measurements were performed on an average P-QRS-T interval. The perioperative ECG changes were different for the DM and non-DM groups. Patients with DM demonstrated a deterioration in several ECG parameters: QRSampl, QRSmorph, Tamp and HR. QRSdur did not change significantly in DM, while it improved perioperatively in non-DM subjects.

Conclusion: ECG parameters respond differently in the perioperative period of $C A B G$ surgery in $D M$ and non-DM patients. Diabetics suffer a deterioration in most of the measured ECG indices, which was not observed in non$D M$.

\section{Introduction}

According to latest clinical recommendations, the most typical patient for surgical coronary revascularization is a diabetic with multivessel disease. Recent data showed improved results of treatment of patients with coronary artery disease (CAD) in presence of diabetes mellitus when surgery - coronary bypass grafting (CABG) is compared to percutaneous coronary intervention (PCI). Publications in literature are showing the benefits of $\mathrm{CABG}$ versus $\mathrm{PCI}$ in terms of repeated revascularizations and survival $[1, \underline{2}]$ especially in diabetic subgroup. Despite advances of both surgery and percutaneous techniques, in latest years patients with diabetes remain still with greater chance for postoperative complications. They are often with small vessel disease and multiple comorbidities: renal impairment, other vascular diseases, etc. [3] . The dynamic of several electrocardiographic (ECG) parameters that are well known risk markers of arrhythmias, incidental heart failure and sudden cardiac death (SCD) could be indicative for pre- and postoperative condition and risk stratification of patients undergoing CABG.

In our previous study on patients undergoing $\mathrm{CABG}[\underline{4}$, 5] we found that QRS and $\mathrm{T}$ wave amplitude and ST segment deviation are not affected by CABG surgery while there is an increase in heart rate (HR), marginal increase in TWA and decrease in QRS-T angle in the early postoperative period.

The severity of vascular changes and the resulting impairment of myocardial perfusion in patients with CAD and diabetes type II (diabetes mellitus, DM) lead to a greater probability for postoperative complications and insufficient revascularization even when surgical revascularization is applied. In the present study ECG parameters in diabetic patients undergoing CABG are compared with nondiabetic, checking the hypothesis that more severe coronary disease could affect postoperative dynamic of these parameters

\section{Materials and methods}

Rest ECG recordings of 50 patients were acquired both before and within the first ten days postoperatively. Our group consisted of 48 men and 2 women. Mean age was 63.2 \pm 7.5 years. Eighteen patients were diagnosed with type II diabetes. Six of these diabetics were on subcutaneous insulin treatment. All patients underwent elective CABG surgery. Patients with recent myocardial infarction (MI), failed PCI and postoperative MI were excluded. CABG was performed on arrested heart using cold blood cardioplegia. The number of bypass-grafts per patient varied from 1 to 4 (47\% of patients received 4 grafts). All patients received at least one arterial bypass with internal thoracic artery (ITA). Postoperatively routine measurement of cardiac specific biochemistry was performed and for all patients in study groups it fell within 
normal range.

Data for blood sugar levels were taken from patients' history and confirmed with baseline blood sugar and glycated hemoglobin. All patients were medicated perioperatively with intravenous rapid acting insulin in order to achieve optimal glucose control during this stressful period and then a stepwise return to preoperative treatment scheme was done.

Signal preprocessing was performed on all recordings to eliminate/suppress the typical noise that accompanies the ECG. The $50 \mathrm{~Hz}$ interference was eliminated by a removal procedure [6], the electromyographic noise was suppressed by dynamic application of an approximation procedure [ $[\underline{7}, \underline{8}]$, and baseline drift was suppressed by a high-pass recursive filter with a cut-off frequency of 0.64 $\mathrm{Hz}[9]$.

Automatic measurement of the ECG parameters was preferred vs. manual one, due to repeatability of the results, lack of bias, and the vast experience of our team in automatic measurement, including:

- $\quad$ QRS detection [10]

- $\quad$ Localization of QRS onset and offset [11]

- $\quad$ Localization of T-wave offset [12]

- QT-interval measurement [13]

- Vectorcardiographic measurements of: 3D angles, surfaces of loops, maximal vectors, etc. $[14,15]$

- T-wave alternans [16]

- $\quad$ QRS alternans [17]
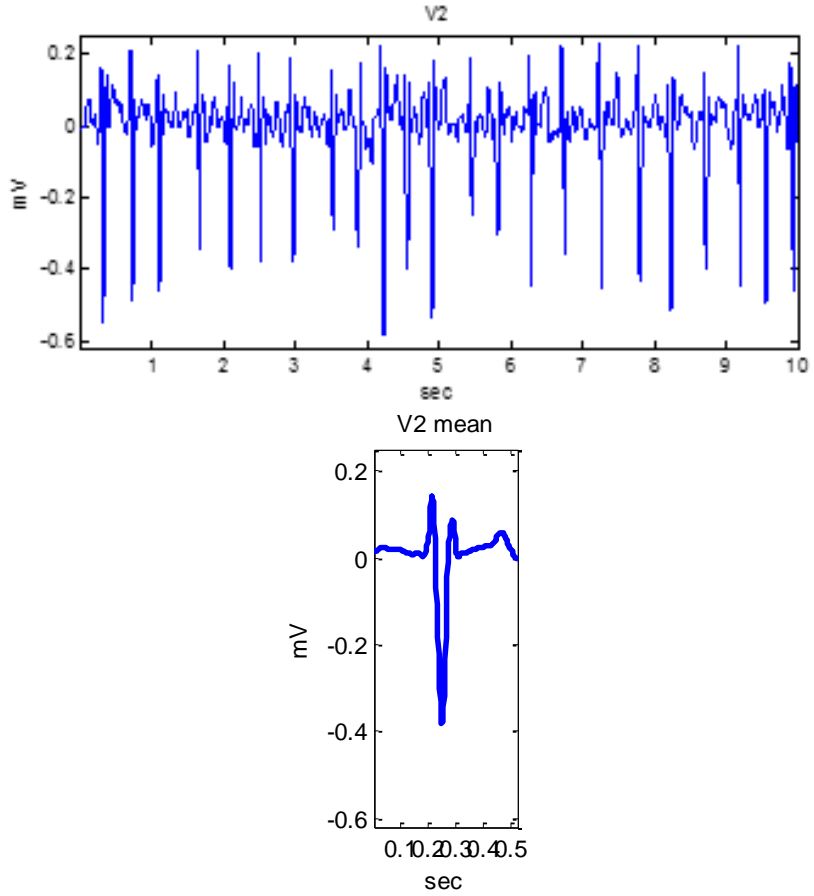

Figure 1. a) V2 lead of a patient with atrial fibrillation; b) P-QRS-T mean value

With automatic parameter's measurement, errors could occur in the presence of noise or at the choice of atypical parameter. For that reason, all ECG parameters were measured on a mean P-QRT-T interval in a certain lead. An example is shown in Fig.1.

All parameters are automatically measured on:

- $\quad$ Precordial V2 lead (amplitudes, durations, STelevations, heart rates),

- $\quad$ Standard 12-leads (QRS and T-wave morphologies, QT-dispersion, QRS alternans, T-wave alternans),

- Computed orthogonal 3D vectorcardiogram (QRS-T angle)

The $\mathrm{X}, \mathrm{Y}$ and $\mathrm{Z}$ leads of the orthogonal vectorcardiogram were computed by the transfer formula of Dewer_1980:

\section{Results}

Results of measurement of the ECG parameters before and after CABG are presented in Table 1.

Table 1. ECG parameters before and after CABG

\begin{tabular}{|c|c|c|c|c|}
\hline & & & \\
& & & \\
& & & \\
& & & \\
& & & & \\
QRSampl & Yes & $1.34 \pm 0.58$ & $1.08 \pm 0.46$ & 0.0018 \\
[mV] & No & $1.04 \pm 0.44$ & $1.13 \pm 0.63$ & n.s. \\
\hline QRSmorph & Yes & $0.24 \pm 0.27$ & $0.14 \pm 0.12$ & 0.026 \\
by PCA & No & $0.21 \pm 0.18$ & $0.26 \pm 0.22$ & n.s. \\
\hline QRSdur & Yes & $101 \pm 11$ & $100 \pm 10$ & n.s. \\
[msec] & No & $120 \pm 28$ & $107 \pm 16$ & 0.006 \\
\hline Tampl & Yes & $0.35 \pm 0.20$ & $25 \pm 0.17$ & 0.05 \\
[mV] & No & $0.32 \pm 0.22$ & $0.23 \pm 0.25$ & 0.013 \\
\hline Tmorph & Yes & $0.18 \pm 0.08$ & $0.23 \pm 0.19$ & n.s. \\
by PCA & No & $0.09 \pm 0.12$ & $0.16 \pm 0.09$ & 0.006 \\
\hline QT disp & Yes & $19.1 \pm 5.3$ & $16.9 \pm 4.7$ & n.s. \\
[msec] & No & $19.3 \pm 6.2$ & $17.2 \pm 5.5$ & n.s. \\
\hline HR & Yes & $70.7 \pm 10.6$ & $82.4 \pm 10.4$ & 0.002 \\
[bpm] & No & $64.5 \pm 7.2$ & $78.1 \pm 12.9$ & 0.0001 \\
\hline
\end{tabular}

The perioperative ECG changes differed for the DM and non-DM groups. Patients with DM demonstrated a deterioration in several ECG parameters: QRS amplitude, QRS morphology, T-wave amplitude and HR. QRS duration did not change significantly in DM, while it improves perioperatively in non-DM subjects. T-wave morphology, QT-dispersion did not differ significantly between groups. 


\section{Discussions}

QRS amplitude. The QRS amplitude change of a certain patient is associated with the change of the volume of extracellular and intravenous fluid. The QRS increase depends on the fluid volume reduction, and vice versa. Most often, such an increase of the QRS is observed, and is a consequence of hemodialysis procedure in people with chronic renal failure [18-20].

Pradeep at al., [21] argue that high volumes of intravenous fluid during cardiac surgery are associated with an increased 90-days' risk of cardiac mortality. Morin et al., [22] have found that the fluid overload with $\geq 5$ liters increases the risk of postoperative complications, compared with insertion of $1-5$ liters $(p<0.001)$.

The diabetics in our study are showing a significant decrease in QRS amplitude while there is a moderate postoperative QRS amplitude increase in non-diabetics. We can speculate that subgroup differences in QRS voltage are due to more complex disease state and presence of comorbidities (incl. renal impairment, even subclinical) in diabetic population, thus leading to slower normalization of fluid balance postoperatively, even with intensified diuretic therapy.

Batchvarov et al., [23] have analyzed the QRS morphology by principal component analysis (PCA ratio of 2nd to 1st eigenvalue) during diagnostic pharmacological (Ajmaline) test for suspected Brugada syndrome. Symptomatic patients with non-diagnostic resting ECGs have increased depolarization heterogeneity. PCA could detect depolarization heterogeneity and thus help the diagnosis and risk stratification of patients with Brugada syndrome. Here in sub group analysis we found a tendency toward decrease of QRS-PCA in diabetics which could be indicative for increased risk for ventricular arrhythmia during the recovery period after surgical intervention in diabetics.

QRS duration is known independent predictor of poor outcomes it is related to increased cardiac mortality [24]. In our study QRS duration is decreasing postoperatively. While in diabetic patients this decrease is virtually absent only $1 \mathrm{~ms}$ in non-diabetic the QRS shortening is average 13 ms, which is found to be statistically significant. In Fig. 3 postoperative reduction of QRS duration is demonstrated. Before the operation it is $171 \mathrm{~ms}$ and after the surgery it comes to $108 \mathrm{~ms}$.

T-wave morphology is very representative in detection of T-wave alternans and risk stratification after myocardial infarction [25]. Breslow at al., [26] have studied the changes of T-wave morphology following anesthesia and surgery. In our subgroup analysis we found tendency toward increase in non-diabetic patients which could be related to improved myocardial perfusion.
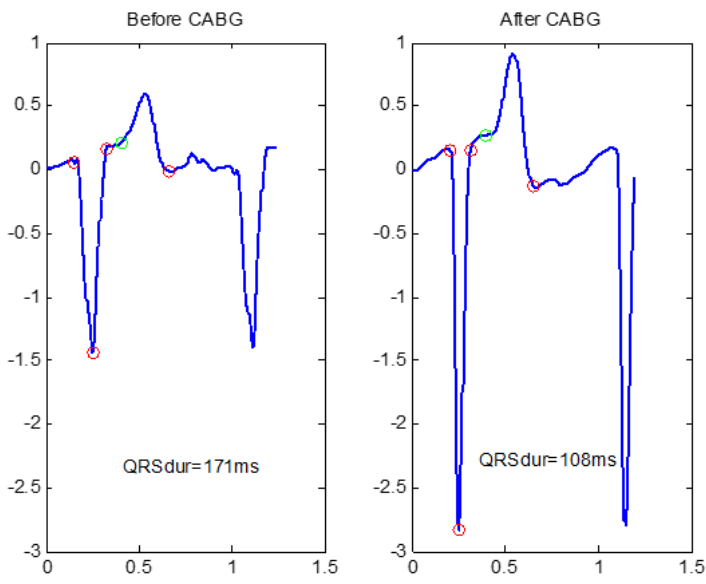

Figure 3. Example $C A B G$ induced shortening of QRS from $171 \mathrm{~ms}$ to $108 \mathrm{~ms}$ after of surgery

Pre-, and post CABG decrease of $\mathbf{T}$-wave amplitude is a sign of low potassium (hypokalemia). In present study, there are no significant changes in T-wave amplitude parameters, although there is tendency toward decreased amplitude in diabetics subgroup not reaching statistically significance. When CABG-induced T-wave amplitude changes were not significant, it means that there is no considerable change of electrolyte concentration, and especially of those electrolytes in the blood that are responsible for the $\mathrm{T}$-wave change - sodium $(\mathrm{Na})$, and potassium (K) [26].

QT-dispersion did not changed significantly in both groups. In our previous study we did found that QTdispersion is decreasing in the immediate postoperative period following CABG [27], but different method for QT measurement was used.

In our study HR is significantly increased after heart operation and more markedly in non-diabetic subgroup. This parameter is influenced by many factors from cardiac and hemodynamic origin but also from general factors as pain, hypoxia hypothermia, etc. Some studies relate increased HR with higher risk for cardiac events [28]. Here we can explain the observed increase of heart rate with postoperative pain and not sufficient level of beta blockade in this early postoperative period. The remarkable increase in non-diabetic patients could be related to more preserved autoregulation in these individuals.

The limitations of the present study were small sample size and short postsurgical follow up.

\section{Conclusion}

Demonstrative changes in ECG are observed following CABG. This study reveals that ECG parameters respond differently in the perioperative period in DM and non-DM patients. Diabetics suffer a deterioration in most of the measured ECG indices, which was not observed in nonDM. The pre- to post-CABG changes of the ECG 
parameters are indicative for increased risk of adverse events in diabetics.

\section{Acknowledgements}

This study is supported by the Bulgarian Scientific Research Fund, grant T02/11

\section{References}

[1] Ko W, Tranbaugh R, Marmur JD, Supino PG, et al. Myocardial Revascularization in New York State: Variations in the PCI-to-CABG Ratio and Their Implications J. Am. Heart Assoc. 2012; 1: e001446.

[2] Taggart DP. Impact of Type 1 and 2 diabetes mellitus on long-term outcomes after CABG. J. Amer. Coll. Cardiol. 2015;65(16):1653-4.

[3] Soubassi LP, Chiras TC, Papadakis ED, et al. Incidence and risk factors of coronary heart disease in elderly patients on chronic hemodialysis. Int. Urol. Nephr. 2006;38:795-800.

[4] Simov D. Electrocardiographic changes in certain cardiovascular physiological and pathological settings. Impact on coronary artery bypass Grafting. Int J Bioautomation, 2016;20(1).

[5] Simov D, Christov I, Bortolan G, Matveev M, Petrov I, Krasteva V (2015) Changes in the electrocardiogram induced by coronary artery bypass grafting. Comp. Cardiol. 2015;1129-1132

[6] Levkov Ch, Mihov G, Ivanov R, Daskalov I, Christov I, Dotsinsky I. Removal of power-line interference from the ECG: A review of the subtraction procedure. Biomed. Eng. Online 2005;4(50)

[7] Christov I, Daskalov IK. Filtering of electromyogram artifacts from the electrocardiogram. Med. Eng. \& Phys. 1999;21:731-6.

[8] Christov I, Neycheva T, Schmid R, Stoyanov T, Abächerli R. Pseudo real-time low-pass filter in ECG, self-adjustable to the frequency spectra of the waves. Med \& Biol Eng \& Comput, 2017, 10 pages,

[9] Daskalov IK, Dotsinsky IA, Christov II. Developments in ECG acquisition preprocessing parameter measurement and recording. IEEE Eng. in Med. \& Biol. 1998;17:50-8.

[10] Christov II. Real time electrocardiogram QRS detection using combined adaptive threshold. Biomed. Eng. Online. 2004;3(1), 28.

[11] Daskalov IK, Christov II. Electrocardiogram signal preprocessing for automatic detection of QRS boundaries, Med. Eng. \& Phys. 1999;21:37-44.

[12] Daskalov IK, Christov II. Automatic detection of the electrocardiogram T-wave end. Med. \& Biol. Eng. \& Comp. 1999;37:348-53.

[13] Christov I, Simova I. Q-onset and T-end delineation: Assessment of the performance of an automated method with the use of a reference database. Physiol Meas. 2007; 28:213-21.

[14] Simova I, Christov I, Kambova L, et al. QRS and T loops area changes during haemodialysis. Comput Cardiol. 2014;41:409-12.

[15] Bortolan G, Bressan M, Christov I. Review on the diagnostic potentials of the T-loop morphology in VCG. Int. J.
Bioautomation. 2009;13(4):55-71.

[16] Bortolan G, Christov I. T-wave alternans detection by a combined method of principal component analysis and $\mathrm{T}$ wave amplitude. Phys Meas. 2012;33:333-42.

[17] Bortolan G, Christov II, Batchvarov VN, Behr ER. QRS\&T wave alternans and beat-to-beat ventricular repolarization variability assessed from 12-lead Holters in patients with suspected Brugada syndrome. Comp. Cardiol. 2009;36:3058

[18] Astan R, Akpinar I, Karan A, et al. The effect of hemodialysis on electrocardiographic parameters. Ann. Noninv. Electrocardiol. 2015;20:253-7.

[19] Christov I, Simov D, Dotsinsky I, Simova I. Increase of electrical impedance following hemodialysis is not the reason for QRS augmentation. Ann. Noninv. Electrocard. 2016;21(2):214.

[20] Saltykova M et al. Increased QRS voltage during dehydrating. Terapevticheskii arkhiv. 2006;794):18-22.

[21] Pradeep A, Rajagopalam S, Kolli HK, et al. High volumes of intravenous fluid during cardiac surgery are associated with increased mortality. HSR Proc. Intensive Care Cardiovasc. Anesth., 2010;2(4): 287-96.

[22] Morin JF, Mistry B, Langlois Y, et al. Fluid overload after coronary artery bypass grafting surgery increases the incidence of post-operative complications. World J. of Cardiovasc. Surg. 2011;1(2): 18-22.

[23] Batchvarov V, Christov I, Bortolan G, Behr E. Principal component analysis of the QRS complex during diagnostic ajmaline test for suspected Brugada syndrome. Comp Cardiol. 2010;37:501-4

[24] Iuliano S, Fisher S,Karasik P, et al. QRS duration and mortality in patients with congestive heart failure. Am Heart J. 2002;143(6):1085-91

[25] Zabel M, Acar B, Klingenheben T, et al. Analysis of 12-lead T-wave morphology for risk stratification after myocardial infarction. Circ. 2000;102(11):1252-7.

[26] Breslow MJ, Miller CF, Parker CD, et al. Changes in T-wave morphology following anesthesia and surgery: a common recovery-room phenomenon. Anastesiology. 1986;64(3):398-402.

[27] Simov D, Simova I, Danov V, Christov I (2007) Effect of coronary artery bypass grafting on QT interval dispersion. The Internet Journal of Thoracic and Cardiovascular Surgery, 10 (1)

[28] Kannel WB, Kannel C, Paffenbarger RS, Cupples LA. Heart rate and cardiovascular mortality: the Framingham Study. Am Heart J. 1987;113(6):1489-94.

Address for correspondence.

Ivaylo Christov

Institute of Biophysics and Biomedical Engineering

Acad, G. Bonchev, blok 105, 1113 Sofia, Bulgaria

Ivaylo.Christov@biomed.bas.bg 